\title{
A Rare Case of Gastric Perforation by Candida albicans: A Case Report
}

\author{
Amber Prasad*, Md Khalid Anjum, Kumari Seema, \\ Ashok Kumar Sharma and Manoj Kumar \\ Department of Microbiology, Rajendra Institute of Medical Sciences (RIMS), Ranchi, \\ Jharkhand, India
}

Keywords

gastrointestinal tract, haemoglobin, ceftriaxone, laparotomy, histopathological examination

Article Info

Accepted:

15 December 2019

Available Online:

20 January 2020

\section{A B S T R A C T}

Gastric perforation due to fungal microorganisms are unusually rare. Most of the cases of gastric perforation results arising out of complications associated with peptic ulcer diseases (PUD), regular use of non-steroidal anti-inflammatory drugs (NSAID) and gastric cancer. Here we are report a case of a 70 years old male who presented with severe abdominal pain in epigastric region without any history of use of NSAIDs, peptic ulcer disease and gastric neoplasm. The patient underwent an emergency exploratory laparotomy and gastric perforation was repaired by modified Graham's repair. The gastric perforation wedge biopsy revealed presence of growth of Candida and its spores and invading pseudo hyphae. Postoperative period was uneventful. He was treated with fluconazole and was discharged from hospital. It is thus necessary, to rule out not only gastric neoplams or ulcer but also opportunistic infection such as Candida infection when upper gastrointestinal perforation is seen in an elderly patient, to significantly reduce the mortality.

\section{Introduction}

Perforation peritonitis is one of the most common presenting complaints in surgical emergency. Candida albicans is a ubiquitous fungus present in healthy individual and is normal commensal of the gastrointestinal tract. Candida infections generally developed in immunocompromised hosts with malignant tumours, diabetes and those patients who have been on long term steroid, non-steroidal antiinflammatory drugs (NSAID) or any immunosuppressant drugs. ${ }^{1}$ Candida infection of gastrointestinal tract is rare as its $\mathrm{pH}$ is low but following long-term use of antacids, Candida infection found in gastric ulcer in healthy person. Gastric perforation caused by Candida albicans is very rare and it is seen mainly in immune compromised and debilitated patients. 


\section{Case History}

A 70 years old male presented in emergency ward with abdominal pain since 5 days with waxing and waning vomiting associated with abdominal distention and constipation. There was no history of hypertension, diabetes or tuberculosis. He was a non-smoker with occasional alcohol intake. On admission, he had the features of septicemia such as paleness, tachyapnea, tachycardia (116 beats/min.), fever and hypotension. On abdominal examination, tenderness and guarding was present all over abdomen with absent liver dullness. The chest X-ray and Xray abdomen in erect view demonstrated gas under right hemidiaphragm. An impression of secondary peritonitis was diagnosed. His preoperative investigation were haemoglobin $10.2 \mathrm{gm} / \mathrm{dl}$, total count was $15,400 /$ cumm with predominance of neutrophil (81\%), serum electrolyte concentration- sodium 138 $\mathrm{mmol} / \mathrm{L}$, potassium $4.1 \mathrm{mmol} / \mathrm{L}$, bicarbonate $20 \mathrm{mmol} / \mathrm{L}$ and serological tests for HIV, hepatitis $\mathrm{B}$ and $\mathrm{C}$ were negative. After resuscitation with intravenous fluids and under the cover of intravenous ceftriaxone and metronidazole, emergency laparotomy was done. A perforation of size $0.5 \mathrm{~cm} \times 0.5 \mathrm{~cm}$ in the antrum of stomach was repaired with modified Graham's repair. A perforation edge biopsy was done and tissue was sent for histopathological examination. 2.5 litres of bilious fluid was aspirated and sent for culture. Histopathological examination revealed the presence of fungal pseudohypahe. (Fig. 1) Periodic Acid Schiff's (PAS) staining showed a positive staining in these pseudo-hyphae suggestive of a Candida infection. Culture of the fluid in Sabouraud's dextrose agar revealed colonies of Candida species (Fig. 2) which was later identified as Candida albicans. (Fig. 3) Bacterial culture was sterile. Postoperative period was uneventful. Patient was eventually discharged on day 10 with advice of anti-fungal fluconzole $150 \mathrm{mg}$ once a week for 8 weeks.

\section{Results and Discussion}

Candida albicans being a ubiquitous fungus is rarely pathogenic in the gastrointestinal tract. In most cases, Candida infection develops as an opportunistic infection in immunocompromised patients. A fungal growth in encouraged by certain $\mathrm{pH}$ level and by the availability of sugar. Therefore, the patient who regularly use antacids and are diabetic are at increased risk. Some reports indicate that $\mathrm{pH}$ of 5-6 in the stomach is suitable for Candida proliferation. In our case, patient had patient had a history of regular use of antacids, which might have been a predisposing factor for gastric perforation associated with Candidal infection.

A study done by Ears et al., found gut mycosis in $4.35 \%$ between the study period 1960$1964{ }^{2}$ A similar study by Tsukamoto et al., reported gut mycosis in $5.9 \%$ between the study period of 1971 to $1983 .{ }^{3}$ In both these cases, the most commonly effected organ was oesophagus followed by stomach, the small intestine and the large intestine. Minoli et al., reported stomach candidiasis in $0.96 \%$ of the upper intestinal endoscopies. ${ }^{4}$

Scotts et al., reported that the disruption of the stomach mucus membrane was sufficient to cause gastric candidiasis. ${ }^{5} \mathrm{On}$ other hand Nelson et al., ${ }^{6}$ and Minoli ${ }^{7}$ et al., reported that some cases of stomach candidiasis were idiopathic. Kamiya et al., reported that Candida secondarily invaded and proliferated in the ulcer base in most cases. ${ }^{8}$

It is difficult to determine whether the gut candidiasis is idiopathic or secondary, because Candida species are part of normal flora of gut in healthy person. 
Fig.1 Histopathological examination showing fungal hyphae (H \& E x 400)

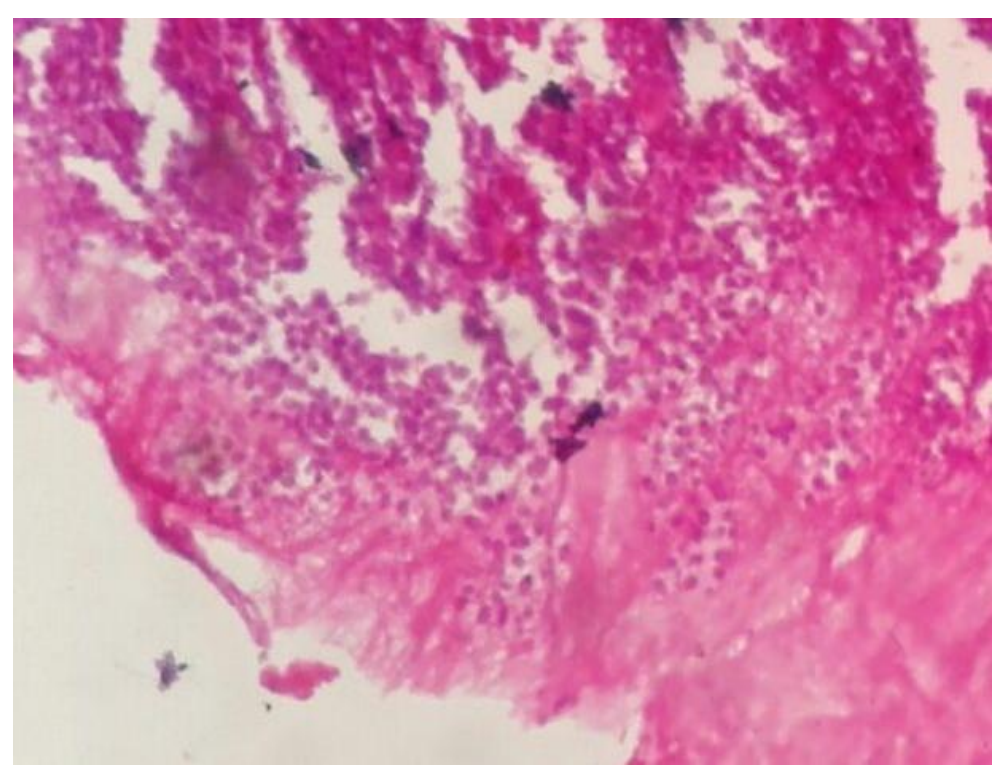

Fig.2 Growth of Candida albicans in Sabouraud dextrose agar (SDA)

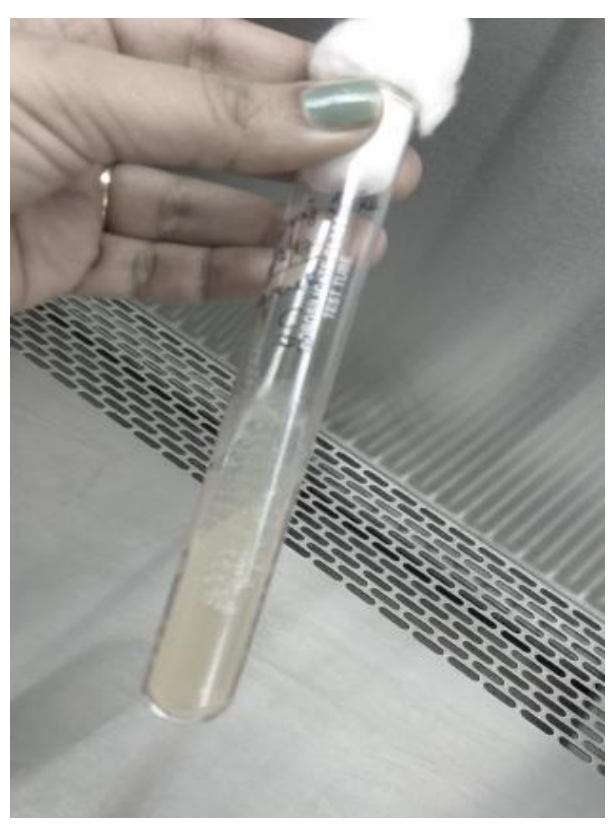


Fig.3 Light microscopy showing fungal hyphae (PAS stain x 40)

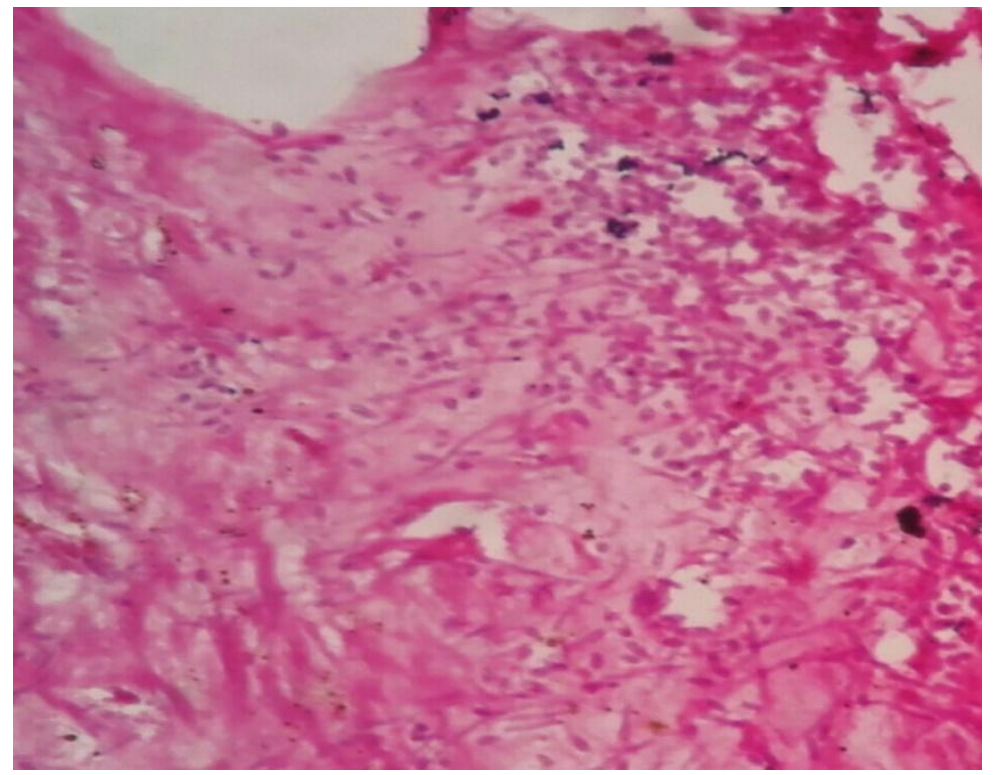

Under normal circumstances, beneficial bacteria control the level of Candida. However, if the bacterial-fungal balance is deranged by usage of antibiotics or if the immunosystem is compromised, an overgrowth of Candida can occur resulting in an infection. ${ }^{9}$

In our case, the patient presented with shock and acute abdominal pain and X-ray revealed gas under right hemidiaphragm suggesting gastrointestinal perforation. An exploratory laparotomy was done which revealed gastric perforation. It was repaired by modified Graham's repair. Postoperative period was uneventful. After biopsy and culture report, tablet fluconazole $150 \mathrm{mg}$ once a week started for 2 months.

Here we experienced a rare case of gastric perforation associated with Candida infection. The fungal infection should always be kept in mind as aetiology for gastric perforation, as early detection is necessary to treat Candida infection. It is thus necessary, to rule out not only gastric neoplams or ulcer but also opportunistic infection such as Candida infection when upper gastrointestinal perforation is seen in an elderly patient, to significantly reduce the mortality.

\section{References}

Bakhshi GD, Borisa AD, Shaikh AS, Thadeshwar NR, Kher Y, Kapadnis LA et al., Invasive gastric candidiasis with perforation. BombayHospital Journal 2011;53(2) :264-65.

Ears P, Goldstein M, Sherlock P. Candida infections of the gastrointestinal tract. Medicine 1972; 51(5): 367-79.

Tsukamoto H. Clinicopathological studies on fungal infections of the digestive tract.Jpn J Gastroenterol 1986;83:2341-50.

Minoli G,Teruzzi V, Butti G, Frigerio G, Rossini A. Gastric candidiasis: an endoscopic and a histological study in 26 patients. Gastrointestinal endoscopy 1982;28:59-61.

Scott BB, Jenkins D. Gastro-oesophageal candidiasis. JAMA 1987;240:21812182.

Nelson RS, Bruni HC, Goldstein HM et al., 
Primary gastric candidiasis in uncompromised subjects. Gastrointest Endosc 1975;22:92-94.

Minoli G, Terruzzi V, Rossini A et al., Gastrointestinal candidiasis occurring without underlying diseases. Endoscopy 1979; 1:18-22.

Kamiya T, Morishita T, Tsutiya M et al., Radiologic and endoscopic features on gastric peptic ulcer associated with Candida infection and its follow-up study. Gastroenterol Endosc 1981;23:1080-1086.

Nalini Gupta A. Rare cause of gastric perforation- Candida infection: A case report and review of the literature. Journal of Clinical and Diagnostic Research. 2012;6 (9):1564-1565.

\section{How to cite this article:}

Amber Prasad, Md Khalid Anjum, Kumari Seema, Ashok Kumar Sharma and Manoj Kumar. 2020. A Rare Case of Gastric Perforation by Candida albicans: A Case Report. Int.J.Curr.Microbiol.App.Sci. 9(01): 119-123. doi: https://doi.org/10.20546/ijcmas.2020.901.013 